\title{
LFV golden channels and effective-field theories
}

\section{Giovanni Marco Pruna*}

INFN, Laboratori Nazionali di Frascati, Via E. Fermi 40, 00044 Frascati (Rome), Italy

E-mail: Giovanni.Marco.Pruna@lnf.infn.it

These proceedings review the status of present and future bounds on muonic lepton flavour violating transitions in the context of an effective-field theory defined below the electroweak scale. A specific focus is set on the phenomenology of $\mu \rightarrow e \gamma, \mu \rightarrow 3 e$ transitions and coherent $\mu \rightarrow e$ nuclear conversion in the light of current and future experiments. Once the experimental limits are recast into bounds at higher scales, the explorative power of such "golden" channels is presented.

The 19th International Workshop on Neutrinos from Accelerators-NUFACT2017

25-30 September, 2017

Uppsala University, Uppsala, Sweden

${ }^{*}$ Speaker. 


\section{Introduction}

Lepton flavour violation (LFV) is strongly suppressed in the framework of the Standard Model (SM) of particle physics. Any observation of such phenomenon in the charged lepton sector would indicate unambiguously the presence of new physics (NP). Therefore, various experimental plans have taken place worldwide to confirm the vanishing prediction of the SM, and investigations are scheduled for the future to explore deeper regions of the NP parameter space.

Remarkable limits have been established with complementary tests of muonic LFV processes by the MEG [1, 2] and SINDRUM [3, 4] collaborations:

$$
\begin{aligned}
& \operatorname{Br}\left(\mu^{+} \rightarrow e^{+} \gamma\right) \leq 4.2 \times 10^{-13}, \\
& \operatorname{Br}\left(\mu^{+} \rightarrow e^{+} e^{-} e^{+}\right) \leq 1.0 \times 10^{-12}, \\
& \operatorname{Br}_{\mu \rightarrow e}^{\mathrm{Au}} \equiv \frac{\Gamma\left(\mu^{-} \mathrm{Au} \rightarrow e^{-} \mathrm{Au}\right)}{\Gamma_{\mathrm{Au}}^{\mathrm{capt}}} \leq 7 \times 10^{-13} .
\end{aligned}
$$

Various experiments are already planned to improve these values by orders of magnitude: the MEG II upgrade [5] with an expected sensitivity of $\operatorname{Br}(\mu \rightarrow e \gamma) \sim 5 \times 10^{-14}$, the Mu3e experiment [6] with an improvement up to four orders of magnitude with respect to SINDRUM, and Mu2e at FNAL and COMET at J-PARC [7, 8, 9] aiming to improve the sensitivity by four orders of magnitude compared with SINDRUM II.

Consequently, these experimental efforts have to be supported both by an accurate theoretical interpretation of possible signals (or absence of signals) in terms of viable NP parameter space and a precise determination of the fundamental backgrounds ${ }^{1}$.

These proceedings explore the possibility to give a model-independent phenomenological interpretation of muonic LFV transitions by adopting an effective-field-theory (EFT) description of NP interactions.

The EFT approach applied to LFV transitions has a long tradition: in the context of neutrino oscillations the first papers were published decades ago [14, 15], while the first complete dimension-six parameterisation at low energy for charged LFV appeared in [16]. On the other hand, the first systematic treatments of charged LFV in the context of SM EFT [17, 18] were published only a couple of years ago [19, 20, 21, 22, 23, 24].

This note summarises the main results obtained in [25, 26], where the EFT parameterisation introduced by [16] was adopted to recast the current and future experimental limits on muonic LFV transitions in terms of bounds on the NP parameter space at the electroweak (EW) energy scale by exploiting a systematic renormalisation-group-equation (RGE) analysis.

\section{Parameterisation}

An effective Lagrangian for the $\mu \rightarrow e$ transitions valid below some scale $\Lambda$ with $m_{W} \geq \Lambda \gg m_{b}$ is considered. It consists of all the operators invariant under $U(1)_{\mathrm{QED}} \times S U(3)_{\mathrm{QCD}}$ and contains all

\footnotetext{
${ }^{1}$ Recent progress has been made in the precise estimation of the $\mu \rightarrow e \gamma[10,11]$ and $\mu \rightarrow 3 e[12,13]$ fundamental background.
} 
the SM fermion fields (except for the top quark) and the QED and QCD gauge fields:

$$
\begin{aligned}
\mathscr{L}_{\mathrm{eff}} & =\mathscr{L}_{\mathrm{QED}}+\mathscr{L}_{\mathrm{QCD}}+\frac{1}{\Lambda^{2}}\left\{C_{L}^{D} O_{L}^{D}+\sum_{f=q, \ell}\left(C_{f f}^{V L L} O_{f f}^{V L L}+C_{f f}^{V L R} O_{f f}^{V L R}+C_{f f}^{S L L} O_{f f}^{S L L}\right)\right. \\
& \left.+\sum_{h=q, \tau}\left(C_{h h}^{T L L} O_{h h}^{T L L}+C_{h h}^{S L R} O_{h h}^{S L R}\right)+L \leftrightarrow R\right\},
\end{aligned}
$$

plus the Hermitian conjugate components wherever required, and the explicit form of the operators given with obvious notation by

$$
\begin{aligned}
O_{L}^{D} & =e m_{\mu}\left(\bar{e} \sigma^{\mu v} P_{L} \mu\right) F_{\mu v}, \\
O_{f f}^{V L L} & =\left(\bar{e} \gamma^{\mu} P_{L} \mu\right)\left(\bar{f} \gamma_{\mu} P_{L} f\right), \\
O_{f f}^{V L R} & =\left(\bar{e} \gamma^{\mu} P_{L} \mu\right)\left(\bar{f} \gamma_{\mu} P_{R} f\right), \\
O_{f f}^{S L L} & =\left(\bar{e} P_{L} \mu\right)\left(\bar{f} P_{L} f\right), \\
O_{h h}^{S L R} & =\left(\bar{e} P_{L} \mu\right)\left(\bar{h} P_{R} h\right), \\
O_{h h}^{T L L} & =\left(\bar{e} \sigma_{\mu v} P_{L} \mu\right)\left(\bar{h} \sigma^{\mu v} P_{L} h\right),
\end{aligned}
$$

with $P_{L / R}=\left(\mathbb{I} \mp \gamma^{5}\right) / 2$. In the equations above, $f$ represents any fermion below the scale $m_{W}$, and $h \in\{u, d, c, s, b, \tau\}$.

In the scenario where NP physics is realised at a scale $\Lambda<m_{W}$, NP gives rise to the interactions described in $\mathscr{L}_{\text {eff. }}$ If BSM physics is beyond the EW scale, $S U(2)$-invariant higher-dimensional operators are generated in the SMEFT. Then, the higher-dimensional operators in $\mathscr{L}_{\text {eff }}$ stem from the matching of the SMEFT to our theory, as performed at the tree level in [27].

In the following analysis, we adopt the RGEs of the operators in $\mathscr{L}_{\text {eff }}$ presented in [26].

\section{Phenomenological results}

The effective coefficients are generated by some underlying NP theory around the EW scale. Then, the RGEs can be exploited to evolve such coefficients from the high scale $m_{W}$ to the phenomenological scales $\mu_{n}$ and $m_{\mu}$. Therefore, the predicted rates are compared with the experimental limits. This procedure will shape the constraints of various Wilson coefficients at the NP scale.

RGE mixing can generate important effects at the phenomenological scale even for vanishing Wilson coefficients at the high scale. This allows one to place bounds on coefficients that would be unconstrained if loop effects are not taken into account.

Considering the experimental limits listed in Section 1, the final aim is to compare the exploratory power of current and future $\mu \rightarrow e \gamma, \mu \rightarrow 3 e$ and $\mu \rightarrow e$ conversion experiments for specific effective interactions at the NP scale.

Here, we assume that at the high scale $m_{W}$ only one Wilson coefficient at a time is non-zero. The corresponding bounds on the coefficients are shown in Table 1 both for the current and for the future experimental limits ${ }^{2}$. We can infer the following general structure of these limits:

\footnotetext{
${ }^{2}$ The best current and future experimental limits are highlighted in green colour.
} 


\begin{tabular}{l||cc|cc|cc}
\multicolumn{1}{l||}{} & \multicolumn{2}{c|}{$\operatorname{Br}\left(\mu^{+} \rightarrow e^{+} \gamma\right)$} & \multicolumn{2}{c|}{$\operatorname{Br}\left(\mu^{+} \rightarrow e^{+} e^{-} e^{+}\right)$} & \multicolumn{2}{c}{$\operatorname{Br}_{\mu \rightarrow e}^{\mathrm{Au} / \mathrm{Al}}$} \\
& $4.2 \cdot 10^{-13}$ & $4.0 \cdot 10^{-14}$ & $1.0 \cdot 10^{-12}$ & $5.0 \cdot 10^{-15}$ & $7.0 \cdot 10^{-13}$ & $1.0 \cdot 10^{-16}$ \\
\hline$C_{L}^{D}$ & $1.0 \cdot 10^{-8}$ & $3.1 \cdot 10^{-9}$ & $2.0 \cdot 10^{-7}$ & $1.4 \cdot 10^{-8}$ & $2.0 \cdot 10^{-7}$ & $2.9 \cdot 10^{-9}$ \\
$C_{e e}^{S L L}$ & $4.8 \cdot 10^{-5}$ & $1.5 \cdot 10^{-5}$ & $8.1 \cdot 10^{-7}$ & $5.8 \cdot 10^{-8}$ & $1.4 \cdot 10^{-3}$ & $2.1 \cdot 10^{-5}$ \\
$C_{\mu \mu}^{S L L}$ & $2.3 \cdot 10^{-7}$ & $7.2 \cdot 10^{-8}$ & $4.6 \cdot 10^{-6}$ & $3.3 \cdot 10^{-7}$ & $7.1 \cdot 10^{-6}$ & $1.0 \cdot 10^{-7}$ \\
$C_{\tau \tau}^{S L L}$ & $1.2 \cdot 10^{-6}$ & $3.7 \cdot 10^{-7}$ & $2.4 \cdot 10^{-5}$ & $1.7 \cdot 10^{-6}$ & $2.4 \cdot 10^{-5}$ & $3.5 \cdot 10^{-7}$ \\
$C_{\tau \tau}^{T L L}$ & $2.9 \cdot 10^{-9}$ & $9.0 \cdot 10^{-10}$ & $5.7 \cdot 10^{-8}$ & $4.1 \cdot 10^{-9}$ & $5.9 \cdot 10^{-8}$ & $8.5 \cdot 10^{-10}$ \\
$C_{b b}^{S L L}$ & $2.8 \cdot 10^{-6}$ & $8.6 \cdot 10^{-7}$ & $5.4 \cdot 10^{-5}$ & $3.8 \cdot 10^{-6}$ & $9.0 \cdot 10^{-7}$ & $1.2 \cdot 10^{-8}$ \\
$C_{b b}^{T L L}$ & $2.1 \cdot 10^{-9}$ & $6.4 \cdot 10^{-10}$ & $4.1 \cdot 10^{-8}$ & $2.9 \cdot 10^{-9}$ & $4.2 \cdot 10^{-8}$ & $6.0 \cdot 10^{-10}$ \\
$C_{e e}^{V R R}$ & $3.0 \cdot 10^{-5}$ & $9.4 \cdot 10^{-6}$ & $2.1 \cdot 10^{-7}$ & $1.5 \cdot 10^{-8}$ & $2.1 \cdot 10^{-6}$ & $3.5 \cdot 10^{-8}$ \\
$C_{\mu \mu}^{V R R}$ & $3.0 \cdot 10^{-5}$ & $9.4 \cdot 10^{-6}$ & $1.6 \cdot 10^{-5}$ & $1.1 \cdot 10^{-6}$ & $2.1 \cdot 10^{-6}$ & $3.5 \cdot 10^{-8}$ \\
$C_{\tau \tau}^{V R R}$ & $1.0 \cdot 10^{-4}$ & $3.2 \cdot 10^{-5}$ & $5.3 \cdot 10^{-5}$ & $3.8 \cdot 10^{-6}$ & $4.8 \cdot 10^{-6}$ & $7.9 \cdot 10^{-8}$ \\
$C_{b b}^{V R R}$ & $3.5 \cdot 10^{-4}$ & $1.1 \cdot 10^{-4}$ & $6.7 \cdot 10^{-5}$ & $4.8 \cdot 10^{-6}$ & $6.0 \cdot 10^{-6}$ & $1.0 \cdot 10^{-7}$ \\
\hline$C_{b b}^{R A}$ & $4.2 \cdot 10^{-4}$ & $1.3 \cdot 10^{-4}$ & $6.5 \cdot 10^{-3}$ & $4.6 \cdot 10^{-4}$ & $1.3 \cdot 10^{-3}$ & $2.2 \cdot 10^{-5}$ \\
$C_{b b}^{R V}$ & $2.1 \cdot 10^{-3}$ & $6.4 \cdot 10^{-4}$ & $6.7 \cdot 10^{-5}$ & $4.7 \cdot 10^{-6}$ & $6.0 \cdot 10^{-6}$ & $1.0 \cdot 10^{-7}$
\end{tabular}

Table 1: Limits on the various coefficients $C_{i}\left(m_{W}\right)$ from current and future experimental constraints, assuming that (at the high scale $m_{W}$ ) only one coefficient at a time is non-vanishing and not including operatordependent efficiency corrections. Green boxes contain the best current and future experimental limits.

- Experimental bounds on the direct $\mu \rightarrow e \gamma$ transition represent a powerful tool to test the Wilson coefficients of the dipole operator. Furthermore, the impact of mixing effects originating from some scalar and tensor operators can also be examined with high precision. However, future prospects for nuclear conversion are so good, that it could overtake the direct $\mu \rightarrow e \gamma$ limits. The only (numerically accidental) exception is represented by $C_{\mu \mu}^{S}$, that will be still better constrained by the next generation of $\mu \rightarrow e \gamma$ experiments.

- A $\mu \rightarrow 3 e$ experiment is the most powerful tool to explore $\mu-e-e-e$ Wilson coefficients of four fermion operators, regardless of the Dirac structure of the operator. This is mainly due to the fact that such interactions produce the $\mu \rightarrow 3 e$ decay already at the tree level while it enters all other processes only via loop effects.

- As expected, $\mu \rightarrow e$ conversion is the most sensitive experimental framework to explore the set of operators including quarks (with exception of current limits on $C_{b b}^{T}$, the Wilson coefficients of tensor operators) and gluons. However, it also appears to be the best setup to study any kind of vector interaction (with the exception of the aforementioned $C_{e e}^{V}$ operators, for which $\mu \rightarrow 3 e$ represents the golden channel). This is mostly due to notable RGE effects in the vector operator mixing matrix.

Concerning $\mu \rightarrow e$ conversion, it is important to keep in mind that, for Wilson coefficients given at the low experimental scale, the $\mu \rightarrow e$ conversion rate is only sensitive to operators with vector or scalar currents on the quark side, but not to operators with axial-vector or pseudo-scalar 
currents. Therefore, it is informative to switch the basis and consider operators with scalar (vector) and pseudo-scalar (axial-vector) currents instead:

$$
\begin{aligned}
C_{f f}^{X S}=\frac{C_{f f}^{S X R}+C_{f f}^{S X L}}{2}, & C_{f f}^{X V}=\frac{C_{f f}^{V X R}+C_{f f}^{V X L}}{2}, \\
C_{f f}^{X P}=\frac{C_{f f}^{S X R}-C_{f f}^{S X L}}{2}, & C_{f f}^{X A}=\frac{C_{f f}^{V X R}-C_{f f}^{V X L}}{2},
\end{aligned}
$$

where $X \in\{L, R\}$ and $f \in\{u, c, d, s, b, e, \mu, \tau\}$. In a simplistic tree-level approach, $\mu \rightarrow e$ conversion is not sensitive to $C_{f f}^{X A}$ and $C_{f f}^{X P}$. However, the remarkable outcome is that axial-vector operators mix into vector operators. This results in strong bounds from $\mu \rightarrow e$ conversion in nuclei once the Wilson coefficients are evaluated at a scale higher than the experimental scale. Therefore, the common preconceptions that $\mu \rightarrow e$ conversion is not sensitive to axial-vector currents is not true anymore once loop effects are taken into account, as one can read from the last rows of Table 1.

\section{Conclusion}

In these proceedings, an RGE-improved analysis of the three $\mu \rightarrow e$ processes $\mu \rightarrow e \gamma, \mu \rightarrow 3 e$ and $\mu \rightarrow e$ conversion in nuclei in the context of EFT was briefly reviewed.

The complete set of dimension-six operators giving rise to point-like muonic LFV interactions invariant under $U(1)_{\mathrm{QED}} \times S U(3)_{\mathrm{QCD}}$ was introduced. Then, the resulting bounds on the Wilson coefficients (given at the scale $m_{W}$ ) were calculated. Afterwards, we stressed the inaccuracy of some opinions that are widely held in the LFV community. Especially, the potential of $\mu \rightarrow e \gamma$ experiments to explore scalar four-fermion interactions and the impact from coherent $\mu \rightarrow e$ conversion on vectorial four-fermion interactions with heavy fermions was clearly displayed.

The present analysis is far from being exhaustive; indeed, intriguing new ideas to explore the effective parameter space appeared recently in the literature [28, 29, 30, 31, 32], and their RGEimproved analysis should be included in the present treatment.

Finally, even if a systematic study was never performed in this direction, we claim that future developments of the $\mu e$-scattering (MUonE) experiment $[33,34]$ could increase our current knowledge of muon LFV transitions and a dedicated EFT analysis should be performed in a future study.

\section{References}

[1] J. Adam et al. (MEG), Phys. Rev. Lett. 110, 201801 (2013), 1303.0754

[2] A.M. Baldini et al. (MEG), Eur. Phys. J. C76, 434 (2016), 1605 . 05081

[3] U. Bellgardt et al. (SINDRUM), Nucl. Phys. B299, 1 (1988)

[4] W.H. Bertl et al. (SINDRUM II), Eur. Phys. J. C47, 337 (2006)

[5] A.M. Baldini et al. (2013), 1301.7225

[6] A. Blondel et al. (2013), 1301.6113

[7] R.M. Carey et al. (Mu2e), FERMILAB-PROPOSAL-0973 (2008) 
[8] R.K. Kutschke, The Mu2e Experiment at Fermilab, in Proceedings, 31st International Conference on Physics in collisions (PIC 2011): Vancouver, Canada, August 28-September 1, 2011 (2011), 1112.0242 , http://inspirehep.net/record/1079590/files/arXiv:1112.0242.pdf

[9] Y.G. Cui et al. (COMET), KEK-2009-10 (2009)

[10] M. Fael, L. Mercolli, M. Passera, JHEP 07, 153 (2015), 1506.03416

[11] G.M. Pruna, A. Signer, Y. Ulrich, Phys. Lett. B772, 452 (2017), 1705 . 03782

[12] M. Fael, C. Greub, JHEP 01, 084 (2017), 1611 . 03726

[13] G.M. Pruna, A. Signer, Y. Ulrich, Phys. Lett. B765, 280 (2017), 1611.03617

[14] S.T. Petcov, Sov. J. Nucl. Phys. 25, 340 (1977), [Erratum: Yad. Fiz.25,1336(1977)]

[15] P. Minkowski, Phys. Lett. 67B, 421 (1977)

[16] Y. Kuno, Y. Okada, Rev. Mod. Phys. 73, 151 (2001), hep-ph/9909265

[17] W. Buchmuller, D. Wyler, Nucl. Phys. B268, 621 (1986)

[18] B. Grzadkowski, M. Iskrzynski, M. Misiak, J. Rosiek, JHEP 10, 085 (2010), 1008 . 4884

[19] A. Crivellin, S. Najjari, J. Rosiek, JHEP 04, 167 (2014), 1312 . 0634

[20] E.E. Jenkins, A.V. Manohar, M. Trott, JHEP 10, 087 (2013), 1308 . 2627

[21] E.E. Jenkins, A.V. Manohar, M. Trott, JHEP 01, 035 (2014), 1310.4838

[22] R. Alonso, E.E. Jenkins, A.V. Manohar, M. Trott, JHEP 04, 159 (2014), 1312 . 2014

[23] G.M. Pruna, A. Signer, JHEP 10, 014 (2014), 1408.3565

[24] G.M. Pruna, A. Signer, EPJ Web Conf. 118, 01031 (2016), 1511.04421

[25] A. Crivellin, S. Davidson, G.M. Pruna, A. Signer, Complementarity in lepton-flavour violating muon decay experiments, in 18th International Workshop on Neutrino Factories and Future Neutrino Facilities Search (NuFact16) Quy Nhon, Vietnam, August 21-27, 2016 (2016), 1611.03409 , http://inspirehep.net/record/1497000/files/arXiv:1611.03409.pdf

[26] A. Crivellin, S. Davidson, G.M. Pruna, A. Signer, JHEP 05, 117 (2017), 1702 . 03020

[27] S. Davidson, Eur. Phys. J. C76, 370 (2016), 1601.07166

[28] M. Koike, Y. Kuno, J. Sato, M. Yamanaka, Phys. Rev. Lett. 105, 121601 (2010), 1003.1578

[29] Y. Uesaka, Y. Kuno, J. Sato, T. Sato, M. Yamanaka, Phys. Rev. D93, 076006 (2016), 1603.01522

[30] Y. Uesaka, Y. Kuno, J. Sato, T. Sato, M. Yamanaka (2017), 1711.08979

[31] V. Cirigliano, S. Davidson, Y. Kuno, Phys. Lett. B771, 242 (2017), 1703.02057

[32] S. Davidson, Y. Kuno, A. Saporta (2017), 1710.06787

[33] C.M. Carloni Calame, M. Passera, L. Trentadue, G. Venanzoni, Phys. Lett. B746, 325 (2015), 1504.02228

[34] G. Abbiendi et al., Eur. Phys. J. C77, 139 (2017), 1609.08987 